\title{
Une étude exploratoire des ponts entre invention, innovation et droits de propriété intellectuelle
}

\author{
An exploratory Study of Bridges between Invention, Innovation \\ and Intellectual Property Rights
}

\author{
Pierre Saulais ${ }^{1}$ \\ ${ }^{1}$ Laboratoire LITEM, Institut Mines-Télécom, Paris, France, pierre.saulais@telecom-em.eu
}

\begin{abstract}
RÉSUMÉ. L'objet du présent article est de compléter les contributions empiriques, déjà fournies pour le numéro spécial de la revue Technologie et Innovation, par un volet plus conceptuel de la thématique des liens entre invention, innovation et droits de propriété intellectuelle. En particulier, après avoir éclairé les trois composantes mentionnées, l'auteur souhaite montrer que ces liens constituent en fait des ponts entre des champs peu reliés jusqu'à présent et que la matière dont ces ponts sont faits n'est autre que la connaissance.

ABSTRACT. Present paper aims at complementing empirical contributions, already delivered to the special issue of Technology and Innovation review, with a more conceptual aspect of the topic of links between invention, innovation and intellectual property rights. After a light exploration of the three above-mentioned components, the author intends to show that these links actually represent bridges between fields which were little connected up to know and that the material, which these bridges are made of, is nothing else that knowledge.

MOTS-CLÉS. Invention, innovation, droits de propriété intellectuelle, patrimoine intellectuel inventif, management des connaissances, innovation fondée sur la connaissance.

KEYWORDS. Invention, innovation, intellectual property rights, inventive intellectual corpus, knowledge management, knowledge-based innovation.
\end{abstract}

\section{Introduction}

«La science et la technique ont véritablement scellé leur union au cours du siècle qui vient de s'achever [...] mais, ce n'est que depuis une vingtaine d'années que les grandes entreprises, poussées à la créativité par la concurrence, se sont rapprochées des universités dès la naissance d'une idée de nouveau produit ou de nouvelle méthode de production. Les universités, confrontées à la réduction des dépenses publiques en $\mathrm{R} \& \mathrm{D}$, ont vu dans la valorisation de la recherche, par les contrats avec les entreprises ou par le dépôt et l'exploitation de brevets, un moyen de financer leurs activités [...] D'un autre côté, le financement public de la recherche a longtemps été justifié par le fait que les connaissances ainsi produites étaient ainsi largement et librement diffusées » (LAPERCHE 2001a). Cet auteur constate ainsi la transformation croissante des créations intellectuelles en marchandises commercialisables et s'intéresse aux rôles de la propriété industrielle dans les différentes étapes de la vie d'une entreprise innovante. Les premiers liens établis entre création technique, droits de propriété industrielle et entreprise innovante sont ainsi essentiellement de nature économique, où seule la propriété industrielle intervient. La réussite d'une invention ne dépend pas que de la créativité de son inventeur, ni du seul dépôt de demande de brevet, mais aussi (et surtout, dans cette perspective économique) d'un ensemble de facteurs économiques, sociaux et politiques qui favorisent ou pénalisent la diffusion des inventions (BOUTILLIER 2001). En complément, Dimitri Uzunidis (2001) focalise son attention sur «la marchéisation de la recherche publique » qu'il voit dans la loi sur l'innovation et la recherche du 12 juillet 1999 (Loi n 99-587), en termes d'appropriation privée des résulta ts de la recherche publique alors que l'objectif de cette loi est «d'intensifier les rapports systémiques entre la recherche publique, l'entrepreneuriat et l'innovation» (UZUNIDIS 2001).

Ceci étant posé, il nous semble qu'en dehors du prisme économique et de son analyse d'effets, peu de travaux académiques ont essayé de rapprocher les domaines de connaissances que représentent 
l'étude des fondements du fait d'inventer, l'étude des fondements du fait d'innover et l'étude des fondements de la propriété juridique (SAULAIS 2013). Notre propos est donc ici de considérer les trois éléments innovation, invention et propriété juridique intellectuelle, non pas comme trois données économiques, mais comme trois objets de connaissance dont il s'agit d'explorer la nature puis les interactions génétiques (au sens de la dynamique de génération).

Le plan de l'article va directement découler de la mention que nous venons de faire de notre propos. Dans une première partie $(\S 1, \S 2, \S 3)$, nous allons nous attacher à la présentation succincte et successive de chacun des trois objets de connaissance, tout d'abord l'acte d'innover et son résultat, puis la capacité à inventer, enfin le patrimoine intellectuel inventif et le capital incorporel (que nous prendrons grand soin de distinguer). La deuxième partie (§ 4) sera dédiée au brossage d'un panorama des liens entre ces trois objets de connaissance, dont la combinatoire vaut six. Ce ne sera qu'un brossage dans la mesure où certains éléments de cette combinatoire sont approfondis de façon spécialisée dans d'autres contributions. Dans une troisième partie ( $(5)$, nous nous interrogerons sur la nature de ces liens au sens où un des objets de connaissance est en mesure d'en stimuler un autre et notre questionnement portera sur une cause possible de cette capacité.

\section{L'innovation}

\subsection{Aspect protéiforme de l'innovation}

Selon Peter Drucker (PRAX, BUISSON et SILBERZAHN 2005), l'innovation consiste en la recherche déterminée et organisée de changement et dans l'analyse systématique des opportunités que de tels changements peuvent offrir en termes de progrès économique ou social : l'innovation est l'acte qui consiste à attribuer à des ressources une nouvelle capacité de créer de la richesse et à en réaliser une diffusion. Une innovation serait ainsi une invention qui a été acceptée par le marché, c'est-à-dire qu'une idée nouvelle produite mais non exploitée, non matérialisée par un produit ou un service resterait seulement une invention. Jean-Yves Prax (PRAX, BUISSON et SILBERZAHN 2005) souligne que l'innovation n'apparaît pas par hasard, mais qu'elle est le fruit d'une capacité du marché à s'approprier de nouveaux usages, en naissant d'une dialectique entre progrès technique et progrès social. L'impact du changement apporté par l'innovation sur l'activité économique constitue une part importante des travaux récents de recherche sur l'économie de l'innovation (GUELLEC 2009).

De très nombreux points de vue permettent d'éclairer le terme innovation. Le concept d'innovation qualifie à la fois un résultat, une offre de produits et / ou de services ou un changement dans un procédé de production (SOPARNOT et STEVENS 2007). Il renvoie à des champs de recherche multiples relevant des domaines de la stratégie de la firme, du marketing ou de la gestion de projet (SOPARNOT et STEVENS 2007). C'est donc bien un polysème, variant en fonction du contexte et qui peut désigner soit le processus de création aboutissant à un nouvel élément ayant une valeur économique et sociale, soit l'adoption de cet élément par une société, soit cet élément lui-même (FERNEZ-WALCH et ROMON 2006). Ces trois acceptions suggèrent que l'innovation n'existe que par rapport à l'entité qui la reçoit, qu'elle implique une diffusion et qu'elle est un acte délibéré, ce qui amène à la définition ci-dessous :

« Nous définissons l'innovation comme un procédé organisationnel délibéré, qui conduit à la proposition et à l'adoption, sur un marché ou à l'intérieur d'une firme, d'un produit (au sens de l'AFNOR) nouveau. Ce procédé permet à une ou plusieurs entreprises d'améliorer leur position stratégique (conquérir ou accroître un pouvoir de marché) et/ou de renforcer leurs compétences et leurs technologies. Le produit nouveau peut être un objet physique, un service, une technologie, une nouvelle compétence ou la combinaison de plusieurs de ces variables. » (FERNEZ-WALCH et ROMON 2006).

Ainsi, l'innovation n'est pas seulement technologique, ni seulement organisationnelle, ni seulement commerciale, mais elle en intègre les différentes dimensions. 


\subsection{Procédures et procédés d'innovation}

\subsubsection{Les procédures et procédés d'innovation selon l'état de l'art industriel}

Une récente étude du Club Gestion des Connaissances ${ }^{1}$ a fait une synthèse de nombreuses méthodes opératoires d'innovation dans les firmes et elle montre que toutes ces méthodes suivent à peu près la même procédure, synthétisée dans la Figure 1 et détaillée dans (LE LOARNE et BIANCO 2009) :

- Le pré-positionnement stratégique suppose l'innovation comme mode de compétition ;

- La génération d'idées s'appuie sur la stimulation de la créativité. La construction et la qualification de concepts consiste à transformer les idées en concepts d'innovation ;

- La sélection des projets d'innovation se fait par le choix du mode de présentation des projets après définition des critères internes de sélection;

- Le déploiement s'appuie sur la protection, la diffusion et la valorisation de l'innovation.

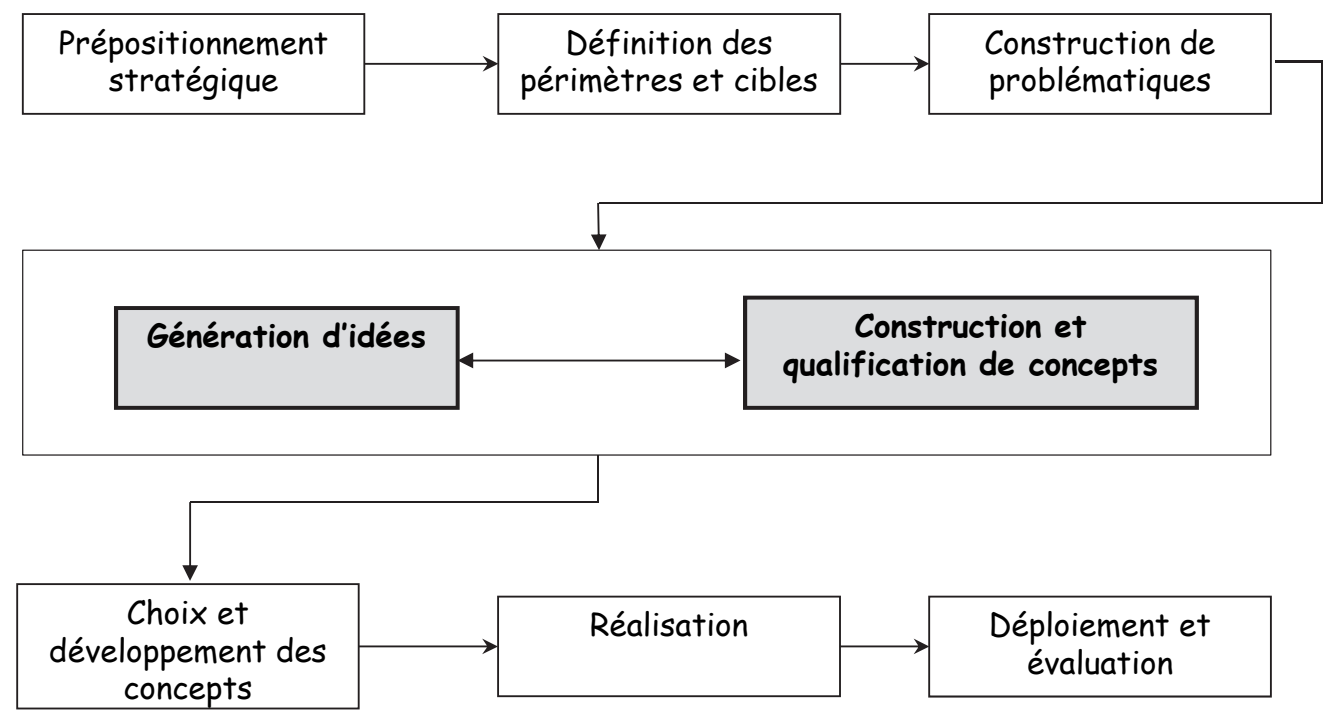

Figure 1. La procédure d'innovation - Source : auteur

Toutes ces méthodes ont en commun la génération d'idées, ce que la partie gauche de la Figure 2 illustre sous l'appellation de « Front End de l'innovation », pour une démarche d'innovation marchande.

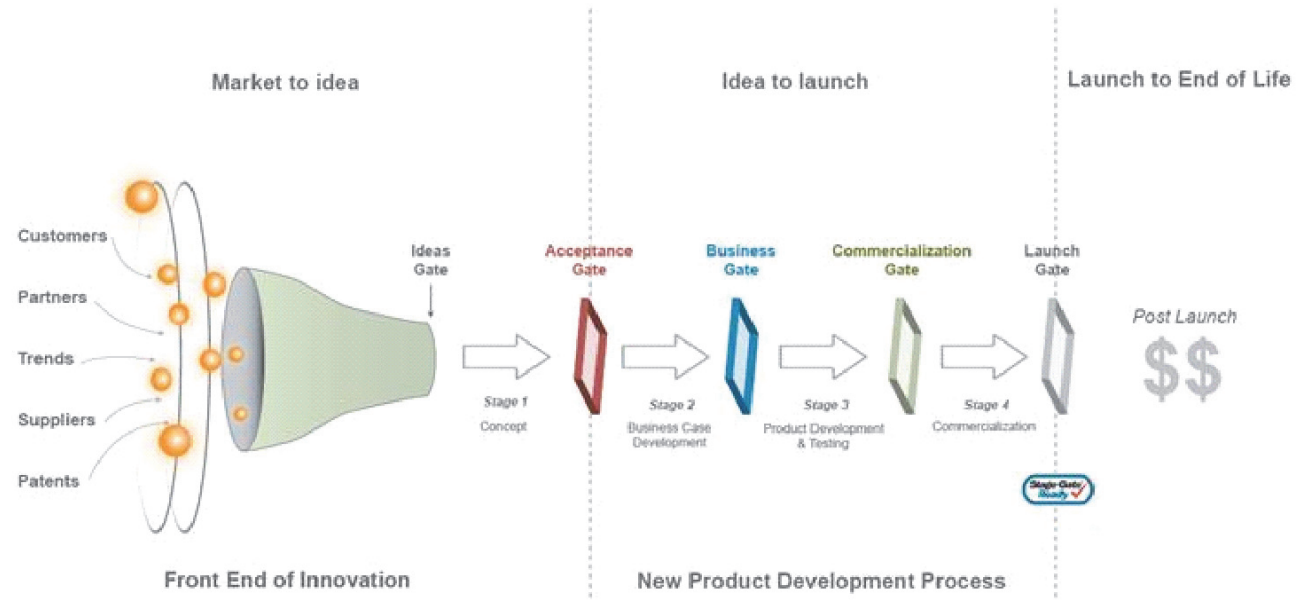

Figure 2. Le Front End de l'innovation - (Source : Club Gestion des Connaissances)

1. Le Club Gestion des Connaissances est une association d'entreprises fondée en 1999 dont l'objectif est de constituer un référentiel commun de concepts et d'outils pragmatiques pour implanter la gestion des connaissances dans les entreprises. 
A ce stade, nous retenons :

- Le concept de changement impliquant un progrès et devant faire l'objet d'une appropriation par le marché ;

- Le résultat, produit ou service ou procédé ou mode d'organisation nouveau, effectivement vendu ou mis en œuvre ;

- Le point focal de la génération d'idée (ou idéation).

\subsubsection{Les procédures et procédés d'innovation selon l'état de l'art académique}

Variété des points de vue sur la procédure d'innovation

La procédure d'innovation est marquée « par un double caractère de récurrence et d'incertitude et son management nécessite vision et créativité » (FERNEZ-WALCH et ROMON 2006). Selon ces auteurs, huit points de vue conceptuels différents peuvent être évoqués sur le déroulement de la procédure d'innovation : valorisation du progrès technique (BIANCHI 1974), adoption d'une nouveauté (ALTER 2000), procédure tourbillonnaire (CALLON et LATOUR 1985), séquence marketing (LAMBIN 1986), transformation d'un système technique (MAFFIN 1998), projet (DECLERCK, EYMERY et CRENER 1980), (NAVARRE et SCHAAN 1989), procédure d'apprentissage collectif (HATCHUEL 1994). La procédure d'innovation peut ainsi représenter « une création collective, organisée dans le temps et l'espace, en vue d'une demande » (GIARD et MIDLER 1993), " une heuristique ouverte qui met aux prises d'une part des individus tendus vers des finalités, projetant des valeurs et des représentations et, d'autre part, un contexte physique et social transformé par l'intervention, mais qui répond, surprend et transforme en retour la trajectoire du concepteur » (GAREL et MIDLER 1995), une procédure de " création de significations et de connaissances nouvelles, qui sont le plus souvent de nature tacite » (CHANAL 1999), une procédure organisationnelle délibérée représentative de situations d'innovation qui nécessitent d'articuler activités d'innovation et activités d'exploitation, avec une mise en cohérence dans le temps selon quatre phases : l'émergence des idées novatrices, la maturation des concepts, le lancement effectif d'un projet innovant, la réalisation du projet (SAULAIS 2013).

\section{Les dimensions multiples de la gestion de la procédure d'innovation}

Peu d'ouvrages traitent d'une manière intégrée des différents aspects de l'innovation (TIDD, BESSANT et PAVITT 2006) : selon ces auteurs, les firmes sont inévitablement limitées dans leur choix de stratégies d'innovation par leurs aptitudes accumulées ainsi que par les opportunités qu'elles sont capables d'exploiter, ce qui veut dire qu'elles se situent sur des trajectoires technologiques.

Une procédure d'innovation globale dans lequel s'inscrit une démarche d'organisation et de gestion des connaissances

Debra Amidon (AMIDON 2001) fonde le pouvoir de l'innovation dans le capital intellectuel et elle considère que ce pouvoir résulte de la conjugaison du partage rapide des connaissances et de l'évolution de nouvelles applications. L'auteur inscrit chacune de ces dimensions dans l'économie des connaissances, où les actifs à gérer sont constitués par la connaissance et par toutes les valeurs immatérielles qui y sont associées et où l'architecture du système de gestion de l'innovation est conçue en vue de l'optimisation de l'utilisation des ressources financières, humaines et techniques : les performances dans l'économie de la connaissance, les structures pour l'exploitation des connaissances, les personnes en tant qu'acteurs de la connaissance, la technologie de traitement des connaissances et la procédure de gestion des connaissances. Ce point de vue est repris par (BENOIT-CERVANTES 2008), selon qui la procédure d'innovation est illustrée par les quatre facettes (Produit, Personnes, Procédures, Potentiel culturel) (BENOIT-CERVANTES 2008) (d'après (Higgins)). 
Les références académiques accordent une place importante à deux points considérés comme clés pour l'innovation : la nécessité du changement et de l'apprentissage. C'est à Gregory Bateson, que revient le mérite d'avoir apporté des éléments déterminants pour la compréhension du processus de changement (BATESON 1972).

Pour Soparnot et Stevens, l'apprentissage organisationnel constitue la caractéristique commune des firmes justifiant de performances sur le long terme, car elles ont développé une capacité à améliorer et à renouveler de façon continue leurs modes de fonctionnement et leurs schémas d'interprétation (SOPARNOT et STEVENS 2007). Il est intéressant de noter que les firmes innovantes sont aussi apprenantes : les projets d'innovation et l'apprentissage organisationnel s'influencent réciproquement. Deux approches complémentaires expliquent les voies de l'apprentissage. Pour March (MARCH 1991), l'apprentissage relève des procédures d'exploitation et d'exploration : l'exploitation peut être synthétisée par la formule « apprendre à faire mieux la même chose » (lien avec l'innovation incrémentale, qui stimule l'exploitation). L'exploration peut être résumée par la formule « apprendre à faire les choses autrement » (lien avec l'innovation de rupture qui stimule l'exploration). Argyris (1993) et Senge (1991) ont développé le point de vue selon lequel une firme est capable de se doter d'une organisation et de mécanismes spécifiques lui permettant de modifier son patrimoine de connaissances . Leur approche est adossée à une position scientifique très précise : la recherche-intervention (ARGYRIS 1993), (SENGE 1991). Les processus d'apprentissage organisationnel sont directement reliés au patrimoine de connaissances et à sa gestion dans la firme.

\subsection{Les étapes intenses en connaissances de la procédure d'innovation}

Nous empruntons au Club Gestion des Connaissances la Figure 3, qui visualise la distribution des huit étapes de la procédure d'innovation en identifiant les étapes où la connaissance est prépondérante.

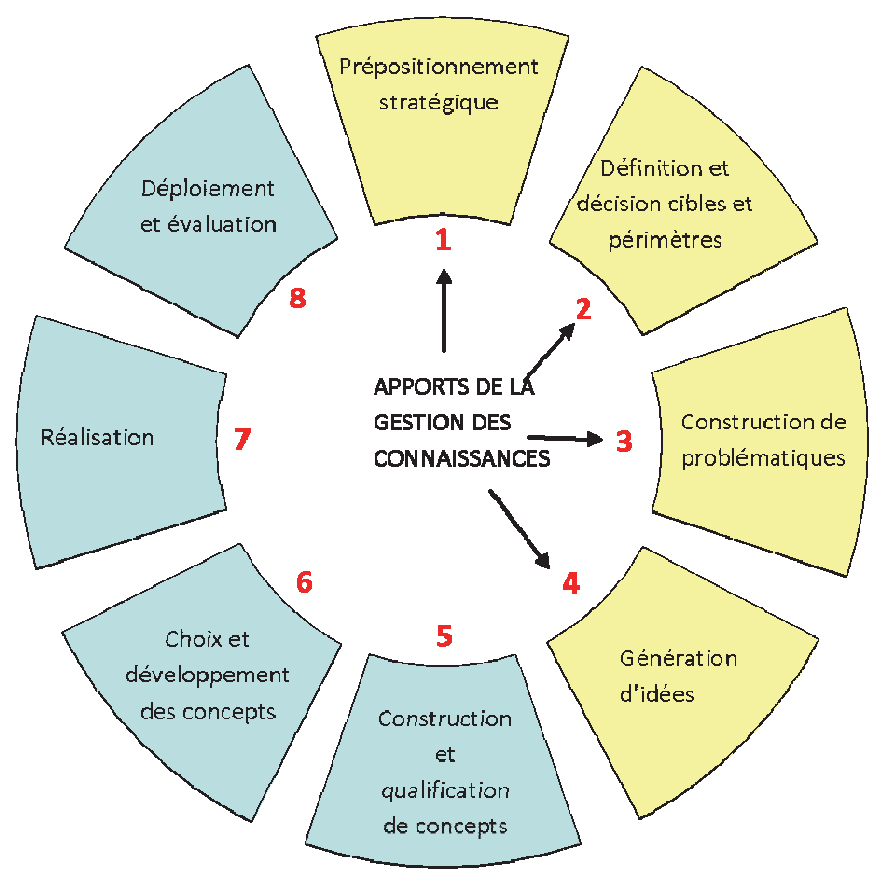

Figure 3. Apport des connaissances à la procédure d'innovation - (Source : Club Gestion des Connaissances)

Les étapes les plus intenses en connaissances sont les quatre premières : le pré-positionnement stratégique, la définition des cibles et des paramètres, la construction de problématiques et la génération d'idées. Ce constat se révèle tout à fait en conformité avec l'intégration notée chez Debra Amidon d'une démarche d'organisation de gestion des connaissances dans la procédure d'innovation. 
En complément, comme le rappellent Soparnot et Stevens, l'innovation contribue elle-même à la création de connaissances de nature différente, qui vont se modifier dans le temps en se combinant et en interagissant entre eux (SOPARNOT et STEVENS 2007). C'est ce que Nonaka et Takeuchi nomment la spirale des connaissances (NONAKA 1997).

Soparnot et Stevens précisent que, si l'innovation contribue à régénérer l'organisation en stimulant la création de connaissances, elle en dépend également, puisqu'elle se développe grâce aux connaissances accumulées dans l'organisation : la gestion de l'innovation appelle donc une gestion des connaissances, puisque, pour partie, les connaissances déterminent l'innovation tout comme elles en proviennent (SOPARNOT et STEVENS 2007).

Tidd, Bessant et Pavitt confirment que l'innovation dépend du savoir en combinant différents ensembles de connaissances (TIDD, BESSANT et PAVITT 2006). Ils font appel pour cela aux travaux de Henderson et Clark (HENDERSON et CLARK 1990), qui ont examiné en détails les types de connaissance impliqués dans différentes catégories d'innovation.

Ainsi, le cœur de la procédure d'innovation (la génération d'idées qui va utiliser la créativité pour constituer un portefeuille d'idées) est bien fortement fondée sur les connaissances.

Cette exploration nous a conforté à la fois dans l'aspect protéiforme de l'approche conceptuelle de la procédure d'innovation nécessitant une vision systémique et dans la place centrale qu'y tient l'idée innovante (tant pour sa génération que pour sa circulation ou la difficulté de son évaluation). Elle nous invite à considérer la nécessité d'intégrer dans la procédure d'innovation une démarche d'organisation et de gestion des connaissances inscrites dans le capital intellectuel et d'en protéger la production par la propriété intellectuelle. La capacité à activer la démarche d'innovation peut être désignée par le terme anglais « innovativeness » (GARCIA et CALANTONE 2001).

\section{L'invention et la capacité à inventer}

\subsection{Notion d'inventivité}

La littérature ne fournit que peu de définitions de l'inventivité (ROUQUETTE 1973), du fait d'une probable confusion avec la créativité. En reprenant le point de vue de l'école de Palo Alto (WATZLAWICK et WEAKLAND 1975), nous dirons que l'inventivité constitue la capacité à modifier les éléments d'un système.

\subsection{Combinaison de la créativité et de l'inventivité}

La créativité correspond à la capacité de susciter un état phénoménal de pensée et l'inventivité est la capacité de mise en action du mécanisme créatif induit par l'état créatif, de façon à produire une création ou œuvre. Créativité et inventivité se combinent donc en une double activité en amont de la procédure d'innovation (SAULAIS 2013).

La procédure d'innovation au sens de génération d'idées est usuellement décrite en quatre étapes (LOUAFA et PERRET 2008) : description de la question, production d'idées divergentes, convergence des idées recueillies vers la question posée, tri et choix.

Les techniques usuelles de production d'idées divergentes visent à la production d'un maximum d'idées dans un temps restreint, en stimulant l'imagination (Table $1 \&$ Table 2). 
La pensée divergente

Suspendre le jugement

Chercher la quantité

Noter toutes les idées

Chercher les idées originales, surprenantes

Combiner et associer les idées

Table 1. La pensée divergente - (Source : auteur)

\begin{tabular}{|c|}
\hline La pensée convergente \\
\hline Juger de façon constructive \\
\hline Améliorer les idées \\
\hline Sélectionner avec des critères \\
\hline Critère : l'inédit \\
\hline Critère : solution adaptée \\
\hline
\end{tabular}

Table 2. La pensée convergente - (Source : auteur)

\subsection{Aperçu de quelques méthodes usuelles}

Dans ce domaine, il y a pléthore de dispositifs classiques : méthode essais/erreurs, Synectique, Questionnaires, Boîtes morphologiques etc. (LOUAFA et PERRET 2008) et de nombreuses techniques : Méthode TRIZ (LOUAFA et PERRET 2008), Thinking Process Approach [approche par les contraintes] (LOUAFA et PERRET 2008), Creative Thinking (SIMON 1969), Lateral Thinking (LOUAFA et PERRET 2008), Brainstorming, Mind mapping (LOUAFA et PERRET 2008), Théorie C-K (HATCHUEL et WEIL 2002) etc.

La procédure globale de résolution de problème décrite dans la Figure 4 vise d'abord à traduire la logique des transitions s'opérant entre les contradictions (LOUAFA et PERRET 2008). Elle débute par la formulation de l'énoncé du problème, qui est progressivement construit au fil de l'examen des divers types de contradictions.

Le terme TRIZ est l'abréviation russe de l'expression « Théorie de résolution inventive de problème », méthode systématique fondée sur la connaissance et tirant ses ressources de la compilation d'un grand nombre de brevets d'invention (LOUAFA et PERRET 2008). TRIZ regroupe un ensemble de méthodes et d'outils, associés à des règles d'utilisation pour déceler des solutions à des problèmes techniques. Genrich Altshuller (ALTSHULLER 1984), (ALTSHULLER 1998). Cette théorie consolidée en 1995 est fondée sur l'idée selon laquelle les découvertes et inventions reposent sur des principes universels. La démarche issue de TRIZ permet notamment de développer de nouvelles solutions à partir des inventions et des connaissances existantes mais externes au champ d'étude. TRIZ peut s'appliquer à la recherche et au développement de nouvelles générations de produits, en proposant un ensemble de lois d'évolution. TRIZ adresse la résolution de problèmes difficiles, avec une méthodologie et des outils s'appuyant sur la résolution des conflits inhérents à tous systèmes techniques (ELTZER et CAVALLUCI 2006). 


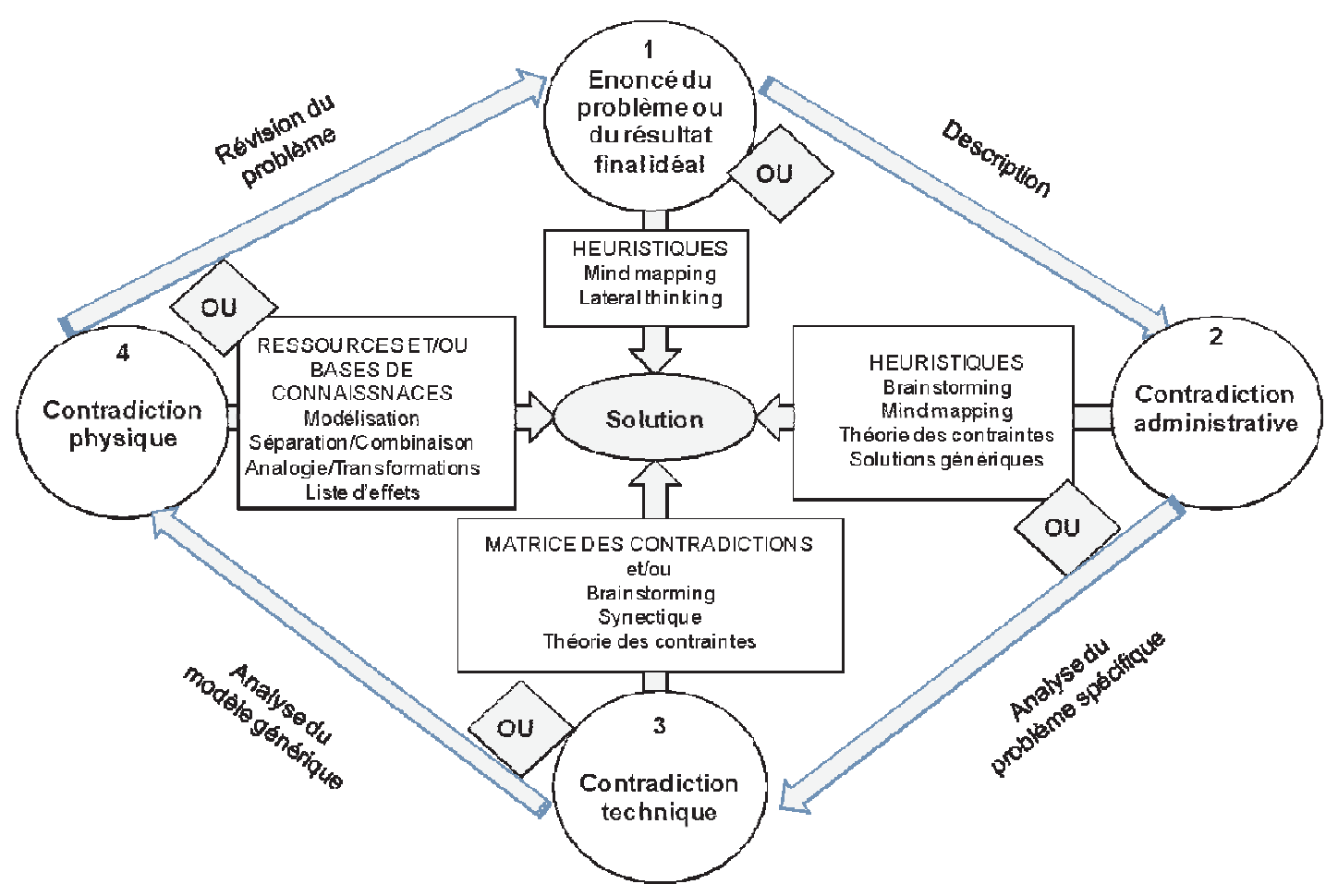

Figure 4. Procédure globale d'intégration des techniques de créativité - Source : Louafa \& Perret, 2008

L'étude systématique des techniques et de leurs fonctions constitue le fondement de TRIZ. Une technique peut exécuter diverses fonctions, parmi lesquelles la fonction primaire qui porte en elle le but de l'existence de la technique. D'autres fonctions auxiliaires accompagnent l'exécution de la fonction primaire, les fonctions utiles, les fonctions nuisibles (produisant des effets indésirables) et les fonctions neutres. Les contradictions constituent le concept central de TRIZ (Cf. Figure 4) et elles sont de trois types, les contradictions administratives (rencontrées quand les directions de recherche de solutions ne sont pas connues a priori), les contradictions techniques (une ou plusieurs directions de recherche de solution émergent qui font faire appel au mode de pensée analogique par le biais de la matrice des contradictions et des 40 principes d'Altshuller) et les contradictions physiques (LOUAFA et PERRET 2008).

\section{Le patrimoine intellectuel inventif}

Nous allons appréhender la nature du patrimoine intellectuel inventif à partir de l'activité de création inventive engendrant une œuvre de l'esprit, sans préjuger de la nature technique ou artistique de la création. Ainsi, nous allons d'abord nous intéresser aux liens entre l'œuvre de l'esprit et le patrimoine intellectuel inventif, puis aux liens entre l'œuvre de l'esprit et le capital incorporel. Ensuite, nous aborderons la description des traces présentes dans le patrimoine intellectuel inventif (traces qui peuvent être représentées par le support matériel de l'œuvre de l'esprit envisagée, puis nous emprunterons la passerelle entre la propriété intellectuelle et la procédure d'innovation. Nous prendrons ensuite le temps d'examiner la question de la valeur du capital incorporel.

\subsection{Cuvre de l'esprit et patrimoine intellectuel}

\subsubsection{Nature du patrimoine intellectuel inventif}

Nous commençons par nous interroger sur la notion de patrimoine : le patrimoine, issu du latin patrimonium (bien de famille), venant lui-même de pater (père de famille, maître de maison) (GAFFIOT 1934) représente, selon la définition d'Emile Littré, un bien d'héritage qui descend, suivant les lois, des père et mère à leurs enfants et qui représente, en général, des biens de famille (LITTRE 1886). Emile Littré note également qu'au figuré le patrimoine évoque "ce qui est considéré comme une propriété patrimoniale » (LITTRE 1886), ce qui, au sens propre, désigne le capital. Selon Gérard Cornu, un patrimoine représente, 
par extension, l'ensemble des biens communément reconnus comme ayant une valeur éminente, par exemple le patrimoine culturel mondial de l'humanité (CORNU 1987). Au sens du patrimoine culturel, le patrimoine suit l'idée d'héritage selon laquelle certains objets du passé doivent être préservés pour être transmis aux générations futures. Le patrimoine est toujours rattaché à un sujet de droit. «Le patrimoine est lié au but qui lui est conféré, voué à la conservation et à la transmission quand il est héritage du passé légué à l'avenir ou aux besoins de son propriétaire quand il est richesse du présent » (ALLAND et RIALS 2003). Notre propos consiste à appréhender la nature du patrimoine intellectuel inventif en tant qu'il est héritage du passé légué à l'avenir, puis à montrer que ce patrimoine abrite un gisement dormant de valeur, dont une analyse structurée par des méthodes d'ingénierie de la connaissance va extraire la richesse du présent.

\subsubsection{Généralités sur les droits de propriété intellectuelle et le droit d'auteur}

La protection par droit d'auteur est accordée dès lors que l'on est en présence d'une création de forme originale, quel que soit le genre auquel elle appartient (littéraire, musical ou artistique), sa destination (documentaire, publicitaire, artistique), sa forme d'expression (orale, écrite, ...) ou le mérite de son auteur. Dans son acception courante, le terme «droits d'auteur» correspond au terme «Propriété littéraire et artistique » dans le code de la Propriété Intellectuelle.

Le Code de la Propriété Intellectuelle dispose que l'auteur d'une œuvre de l'esprit jouit sur cette œuvre, du seul fait de sa création, d'un droit de propriété incorporelle exclusif et opposable à tous, qui comporte des attributs d'ordre intellectuel et moral ainsi que des attributs d'ordre patrimonial (Article L 111-1). Cette propriété incorporelle est, par essence, distincte de la propriété corporelle du support physique de l'œuvre de l'esprit. La propriété incorporelle se rapporte au contenu de l'œuvre, par essence dématérialisé et inaliénable du fait de sa nature purement intellectuelle. La propriété corporelle se rapporte au support de l'œuvre, qui appartient au monde sensible, qui peut être diffusé et faire l'objet de toutes sortes de transaction et qui participe du système d'information, considéré ici comme dépositaire de supports matérialisant la création d'une œuvre de l'esprit.

Le droit de propriété intellectuelle sur une œuvre s'acquiert sans aucune formalité de dépôt, du seul fait de sa création : c'est donc un droit sui generis, c'est-à-dire caractéristique de son espèce (BLOCH et vonWARTBURG 1932). Il confère des droits patrimoniaux (droit de reproduction et de représentation) et des droits moraux (notamment droit au nom et droit au respect de l'œuvre).

Le droit d'auteur est le droit des créateurs : bien que l'on donne l'appellation de propriété littéraire et artistique au droit d'auteur, il ne s'agit pas par là d'accorder aux créateurs une propriété au sens du code civil, mais seulement de les investir d'un privilège exclusif d'exploitation temporaire.

\subsubsection{Représentation du patrimoine intellectuel inventif}

Le point de départ de notre analyse de la nature du patrimoine intellectuel inventif est constitué par l'activité de création inventive, qui correspond à la phase d'effectuation du phénomène de création à double facette, créativité et inventivité. Le produit de cette activité (l'œuvre de l'esprit) ne va engendrer de droits de propriété intellectuelle que si cette œuvre satisfait à des conditions d'originalité qui constituent précisément la signature de la création inventive : cette œuvre va enrichir un corpus que nous allons nommer le patrimoine intellectuel inventif. Comme une caractérisation a posteriori de la nature de l'œuvre peut être obtenue par la satisfaction des critères d'attribution de droits de propriété intellectuelle, ces derniers vont donc constituer des matériaux précieux pour appréhender la nature du patrimoine intellectuel inventif. La création intellectuelle caractéristique de l'œuvre de l'esprit va se traduire en nouvelles connaissances et ce sont ces nouvelles connaissances, qui, après formalisation, vont venir grossir un corpus intellectuel constituant le patrimoine intellectuel inventif, selon un point de vue dynamique. 
Toute œuvre de l'esprit peut engendrer des droits de propriété intellectuelle, traditionnellement séparés en droits de propriété industrielle (notamment les brevets industriels) et droits d'auteur (notamment d'œuvres littéraires et artistiques).

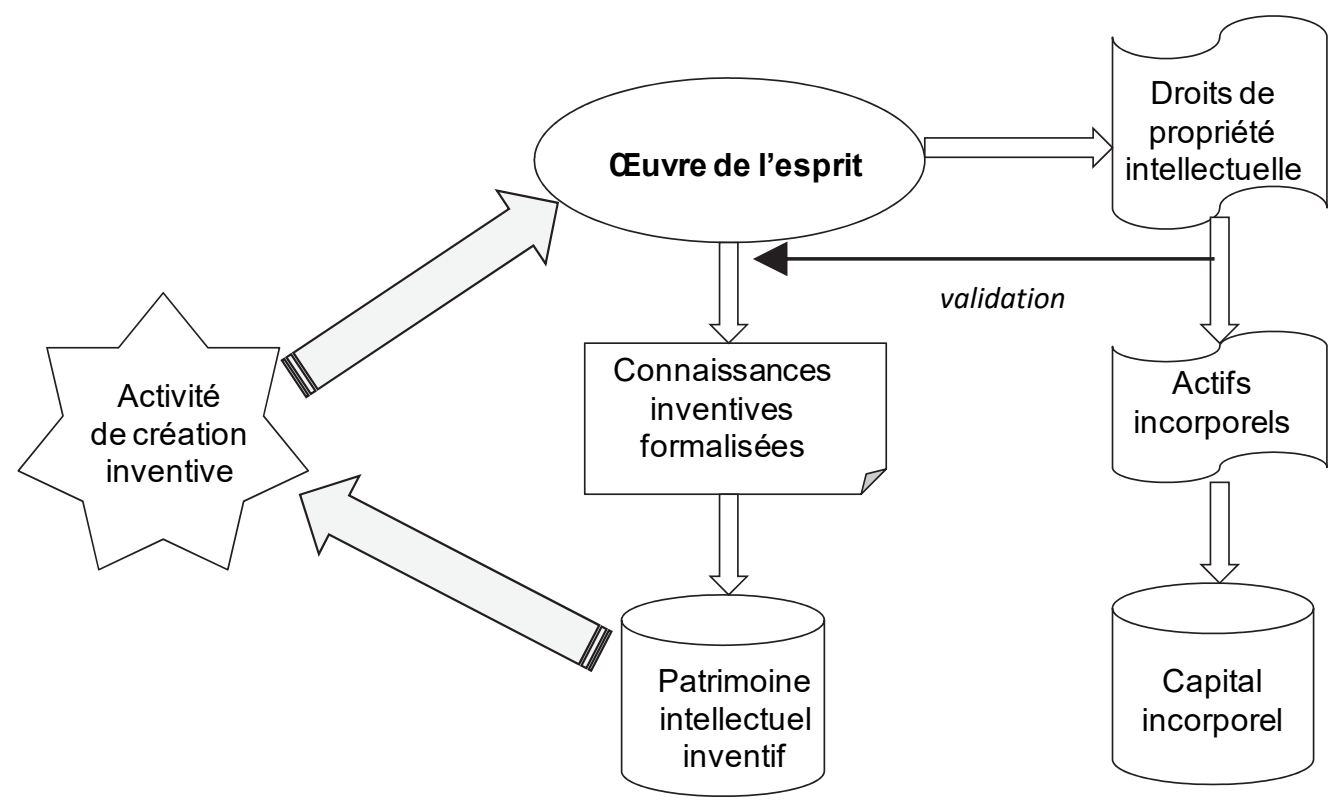

Figure 5. Le Patrimoine intellectuel inventif - Source : auteur

Comme illustré par la Figure 5, l'activité de création inventive constitue le deus ex machina qui engendre l'œuvre de l'esprit (SAULAIS 2013). La reconnaissance de cette œuvre comme originale (pour le domaine artistique et littéraire) ou comme nouvelle et inventive (pour le domaine technologique), permet, dans le cadre du domaine technologique qui nous intéresse ici, de valider la création intellectuelle caractéristique de l'œuvre de l'esprit. Cette création intellectuelle va s'exprimer en nouvelles connaissances, qui, après formalisation, vont venir dynamiquement grossir l'enveloppe du corpus intellectuel constituant le patrimoine intellectuel inventif. Nous avons observé comment la création intellectuelle inventive, en prenant place dans le patrimoine intellectuel inventif (via les nouvelles connaissances qu'elle exprime), en définit la nature et en suggère la méthode d'analyse. Le patrimoine intellectuel inventif n'est donc autre que le dépôt dématérialisé de ces nouvelles connaissances venant féconder les précédentes connaissances accumulées dans le corpus existant, à la fois héritage du passé légué à l'avenir et richesse du présent.

La caractérisation de la nature de l'œuvre peut être obtenue a posteriori par la satisfaction des critères d'attribution de droits de propriété intellectuelle, qui vont consacrer le créateur en tant qu'auteur reconnu juridiquement et économiquement. L'étape suivante de notre cheminement consiste à explorer comment les droits de propriété intellectuelle, reconnaissant une œuvre de l'esprit inventive, vont engendrer un capital incorporel.

\subsection{Cuvre de l'esprit et capital incorporel}

Nous continuons notre analyse de la Figure 5, pour aborder le domaine du capital. Nous commencerons par nous interroger sur la déroutante profusion d'épithètes attribués au capital dans la littérature : intangible, intellectuel, immatériel, incorporel. Ayant jeté notre dévolu sur l'incorporel, nous lèverons successivement le voile sur les biens incorporels, sur les actifs incorporels et enfin sur le capital incorporel.

\subsubsection{Capital intellectuel, immatériel, incorporel}

\subsubsection{Capital intellectuel}

«Intellectual capital is intellectual property in action » (KEEN 1999).

La notion de capital intellectuel se révèle très variable selon les auteurs (BINCTIN 2007). Nicolas Binctin note que l'expression a fait son apparition en juin 1991 dans le magazine Fortune dans un article 
de Stewart (STEWART, Brainpower: How Intellectual Capital is Becoming America's Most Valuable Asset 1991). Le concept de capital intellectuel a suscité plusieurs publications pour les gestionnaires de sociétés (EDVISSON 1997), (BONTIS 1998), (STEWART, Intellectual Capital: The new Wealth of Organizations 1997), (ROOS, et al. 1998) ainsi que des publications universitaires (BONTIS, Managing Organizational Knowledge by Diagnosing Intellectual Capital: Framing and Advancing the State of the Field 1999). Si ces sources permettent de constater une très grande hétérogénéité des éléments regroupés par les sciences de gestion dans le concept de capital intellectuel, elles permettent de noter que trois éléments sont systématiquement abordés par les auteurs : le capital humain, le capital structurel et la propriété intellectuelle :

- Le capital humain ne peut pas être approprié par l'organisation, car il combine les savoirs, l'habileté et les talents des collaborateurs ;

- Le capital structurel regroupe les savoirs hors du capital humain (organisation interne, recueils de procédures...) qui ne sont pas appropriables (sauf pour des éléments spécifiques comme des bases de données) ;

- Les propriétés intellectuelles activent des mécanismes d'appropriation selon plusieurs régimes (propriété industrielle, droit d'auteur...).

Selon Nicolas Binctin, les gestionnaires désignent le capital intellectuel comme l'ensemble des potentialités incorporelles d'une firme non inscrites en compte et ne faisant pas l'objet d'une valorisation spécifique par les documents comptables. Toutefois, en ne retenant que les éléments appropriables, ce capital intellectuel vu par les sciences de gestion se réduit aux créations appropriées par l'un des régimes de propriété intellectuelle (principalement propriété industrielle et droit d'auteur) ou par le secret (BINCTIN 2007).

\subsubsection{Capital immatériel}

Le capital immatériel est une notion couramment utilisée aujourd'hui pour désigner l'ensemble des éléments incorporels dont la possession est susceptible d'apporter à une firme un avantage économique sur son marché (LAPERCHE 2001a), (BOUNFOUR, Capital immatériel, connaissance et performance 2006), (BOUNFOUR et EPINETTE, Valeur et performance des Systèmes d'Information 2006). Ces éléments peuvent être ainsi interprétés comme des actifs incorporels selon (BREESE et KAISER 2004a). Dès lors, la logique voudrait que l'on désignât ce capital comme capital incorporel (SAULAIS 2013), ce qui constituera notre choix de vocabulaire, comme indiqué plus bas.

Michel Vivant et Jean-Michel Bruguière relèvent que « La propriété intellectuelle occupe une place centrale dans l'économie de l'immatériel. En effet, dans une économie où les idées priment, où c'est l'innovation qui crée la valeur, il est normal que les acteurs cherchent à protéger ces idées ou, à tout le moins, le bénéfice économique qu'ils peuvent en tirer » (VIVANT et BRUGUIERE 2009). Selon ces auteurs, le droit d'auteur est « un droit de l'auteur sur l'idée que l'œuvre n'est jamais que la continuation de la personne ». Il en découle que l'auteur occupe une place prééminente et qu'il se voit reconnaître des droits moraux propres à assurer le respect de sa personne comme de son œuvre, puisque l'une et l'autre ne font qu'un.

Pour Blandine Laperche, la notion de capital «immatériel » est aujourd'hui couramment utilisée pour désigner l'ensemble des éléments incorporels dont la possession est susceptible d'apporter à une firme un avantage économique sur son marché (LAPERCHE 2001a). C'est ainsi que Blandine Laperche distingue trois différentes dimensions du capital « immatériel » de la firme innovante :

- Les actifs technologiques ;

- Les éléments ayant trait aux techniques commerciales et aux modes d'accès à la clientèle ;

- L'organisation et les hommes qui la composent. Certains auteurs n'hésitent pas d'ailleurs à évoquer un « capital organisationnel» (LAMBERTON 1997). 


\subsubsection{Capital incorporel}

Notre propos est ici d'éclairer l'apport de l'épithète incorporel à l'actif (actif incorporel), puis au capital (capital incorporel).

Les œuvres de l'esprit ouvrent des droits de propriété intellectuelle, qui constituent une partie des actifs incorporels. Selon Patrick Ochs, un actif incorporel est un investissement dématérialisé, dynamique qui incorpore de manière durable une part de connaissance dominante à fin de contribuer de manière spécifique ou processuelle à la compétitivité et à la valeur de l'entreprise (OCHS 1996). Breesé et Kaiser définissent les actifs incorporels comme suit : " Le terme d'actif incorporel désigne un actif qui tire sa valeur d'une existence non corporelle, c'est-à-dire qu'on ne peut pas le toucher ni le voir, à l'inverse d'un actif corporel qui tire sa valeur de son existence matérielle et de ses propriétés physiques » (BREESE et KAISER 2004a). Ces auteurs notent que la classification des actifs incorporels diffère selon les auteurs. Par exemple, F. Reilly et P. Schweihs proposent la classification suivante (REILLY et SCHWEIHS 2001) : les actifs de marketing (marques, logos, noms commerciaux), les actifs technologiques (brevets, cahiers de laboratoire, savoir-faire), les actifs artistiques (œuvres littéraires, dessins et modèles), les actifs logiciels (bases de données, programmes informatiques, circuits intégrés), les actifs de type ingénierie (design industriel, savoir-faire, secrets, dessins et schémas industriels, documentations techniques), les actifs de clientèle (listes de clients, contrats, relations avec la clientèle), les actifs de contrats (contrats de fourniture, de licence, de franchise, de non concurrence), le capital humain (employés formés et organisés, contrats des salariés), les licences officielles (droits d'exploitation minière, droits de concession sur l'eau, lignes aériennes), le goodwill (célébrité, renommée). Ainsi, nous pouvons distinguer, d'une part, les actifs incorporels formalisés issus d'une œuvre de l'esprit ayant ouvert à droits de propriété enregistrés formellement (droits de propriété industrielle) ou non (droits de propriété littéraire et artistique à titre de droits patrimoniaux et moraux d'auteur) et, d'autre part, des actifs incorporels non formalisés tacites non appropriables, en l'état, par l'entité.

Fort de cette analyse des actifs incorporels, nous pouvons circonscrire logiquement, mais non exhaustivement, le capital incorporel comme ensemble des actifs incorporels.

\subsection{Traces dans le patrimoine intellectuel inventif}

Nous avons défini le patrimoine intellectuel inventif à partir de l'enveloppe incorporelle d'un corpus (BLOCH et vonWARTBURG 1932), (DAUZAT 1971) incluant tous les contenus des œuvres issues de l'activité de création, exclusivement de leur support matériel. Comme mentionné plus haut, corpus est ici pris dans son acception d'ensemble (de textes, de documents) appliqué à leur seul contenu (Cf. corpus : réunion de personnes soumises aux mêmes règles, d'où : recueil de droits ou d'inscriptions, d'après corpus juris). Le patrimoine intellectuel à transmettre n'est pas constitué par des supports matériels, mais par leur contenu : pendant longtemps, la transmission de ce patrimoine intellectuel n'a été qu'orale, mais c'est bien un contenu qui était transmis de génération en génération. Aujourd'hui, ce patrimoine (qui s'accroît sans cesse) est bien trop volumineux pour être transmis oralement.

En pratique, nous allons définir les traces du patrimoine intellectuel inventif comme les marques laissées par les supports matériels transcrivant l'activité inventive, regroupés dans le jumeau corporel de l'enveloppe incorporelle du corpus du patrimoine intellectuel inventif. Dans le cadre de l'organisation, nous allons définir ce jumeau corporel, non exhaustivement, comme l'ensemble des brevets d'invention, articles et communications, rapports d'étude, mémoires de thèse ou de stage, mémoires internes, papiers blancs, présentations, supports de formation, ..., sous réserve qu'ils décrivent une activité intellectuelle inventive.

\subsubsection{Définition du brevet d'invention}

C'est un titre de propriété industrielle qui confère à son titulaire (personne physique ou morale) un monopole d'exploitation sur une invention technique pour une durée maximale de 20 ans ainsi que le 
droit d'interdire à tout tiers non autorisé la reproduction de l'invention telle qu'elle est définie dans les revendications du brevet. Plus que de propriété industrielle, il conviendrait de parler de propriété juridique.

\subsubsection{Notion de brevetabilité}

Une invention technique, c'est-à-dire une innovation technologique à l'origine d'une solution technique apportée à un problème technique donné, n'est brevetable que si elle remplit les trois conditions cumulatives suivantes : application industrielle, nouveauté, activité inventive.

Il ressort que l'invention doit être une création (et non une découverte) qui poursuit un but technique (et non esthétique) et qui doit être finalisée (et non pas une simple idée ou une théorie). Cette invention relève donc bien du domaine de la propriété industrielle (et non pas de la propriété littéraire ou artistique). La notion de brevetabilité est très puissante, puisqu'elle implique à la fois la nouveauté et l'inventivité.

\subsubsection{Information contenue dans un brevet d'invention}

Selon l'Institut National de la Propriété Industrielle (INPI), les 35 millions de brevets d'invention mondiaux représentent $80 \%$ de l'information scientifique et technique et l'OCDE estime que $70 \%$ de l'information contenue dans les brevets d'invention ne se trouve que dans les brevets d'invention. L'information est classée finement selon 70000 classes et elle bénéficie de deux qualités spécifiques essentielles : un plan de rédaction normalisé et adapté à l'innovation et des liens qui facilitent la traçabilité des innovations (BREESE et deKERMADEC 2004b). Par leurs caractéristiques, les bases de brevets d'invention constituent le plus grand réseau mondial de gestion des connaissances.

\subsection{Synthèse sur le patrimoine intellectuel inventif}

Nous avons noté que, à partir d'une œuvre de l'esprit, fruit de l'activité de création, la propriété industrielle engendre le capital incorporel en créant une valeur permettant d'associer, à l'œuvre dématérialisée, un droit susceptible d'appropriation, ayant la nature d'un bien et que ce double titre amène à considérer comme un objet d'échange. La propriété intellectuelle, avec son droit de l'auteur, consacre le créateur comme un auteur (dont l'œuvre est considérée comme la continuation) et valide les nouvelles connaissances qui vont venir enrichir l'enveloppe incorporelle du corpus intellectuel constituant le patrimoine intellectuel inventif.

\section{Analyse des liens entre invention, innovation et patrimoine intellectuel inventif}

\subsection{Liens des droits de propriété industrielle avec l'innovation}

Notre propos est ici de montrer l'apport de l'information protégée par des droits de propriété industrielle à l'innovation.

Blandine Laperche note que le brevet d'invention, dont on comptait en 2000 près de quatre millions de titres actifs dans le monde, (dont 83 \% déposés aux Etats-Unis, en Europe et au Japon ), est devenu un moyen puissant d'incitation à l'invention, du fait de la reconnaissance du rôle des avancées technologiques dans la stimulation de la croissance économique (LAPERCHE 2001b) : le monopole exclusif d'exploitation temporaire qu'accorde le titre de propriété devient le pendant de l'innovation, ou selon la définition de Joseph Schumpeter, de « l'exploitation marchande de l'invention » (SCHUMPETER 1947). En effet, sans protection légale, la nouvelle technique ou le nouveau procédé risquent d'être récupérés et copiés lors de leur diffusion sur le marché. L'octroi d'un monopole d'exploitation temporaire permet à son titulaire de pouvoir récupérer les capitaux qu'il a investis pour donner le jour à son invention, en permettant d'interdire à des concurrents moins créatifs de reprendre sans effort ces innovations, mais aussi en constituant des actifs incorporels qui contribuent à sa valorisation financière ainsi qu'à la valorisation de son image (BREESE et deKERMADEC 2004b). Il s'agit alors d'organiser les relations 
entre les acteurs économiques (franchises, contrats de licence de brevets, ...) et de protéger ses droits dans le respect de ceux des autres (vérification de la liberté d'exploitation). Ainsi, Blandine Laperche note que « les grandes entreprises cherchent aujourd'hui à protéger le mieux possible leur potentiel d'innovation, constitué par des informations, du savoir, du savoir-faire, des techniques et des marchandises constituées ou en cours de constitution » (LAPERCHE 2001b).

A l'heure où chacun s'accorde à dire que la compétitivité des entreprises dépend aujourd'hui principalement de leur capacité à incorporer de nouveaux savoirs dans des produits et services créateurs de valeur, le rôle stratégique des brevets d'invention est difficilement contestable (CORBEL 2007). Toutefois, les savoirs explicites, objet de la protection juridique offerte par les droits de propriété intellectuelle, ne constituent qu'une partie des savoirs mobilisés dans la lute concurrentielle (BAUMARD 1996).

En résumé, le lien entre la propriété industrielle et l'innovation est principalement porté par le lien vers la procédure d'innovation et il est caractérisé par le fait que le rôle protecteur de la propriété industrielle est un puissant incitateur à l'innovation du fait de son droit de monopole d'exploitation.

\subsection{Liens des droits de propriété industrielle avec l'invention}

Notre propos est ici de montrer l'apport de l'information protégée par des droits de propriété industrielle à la capacité d'invention, que ce soit directement (apport informationnel) ou indirectement (apport méthodologique).

\subsubsection{Apport direct des brevets d'invention}

Les brevets publiés aident les chercheurs à explorer de nouveaux domaines (connaissance de ce qui existe et de ce qui est protégé, compréhension des étapes du passé et humilité face à l'avenir, description de l'art antérieur et traçabilité des choix de conception technique : les brevets et les publications sont complémentaires. Les publications sont centrées sur l'explication des phénomènes tandis que les brevets sont positionnés plus en aval, sur les résultats, les applications et les moyens utilisés pour ces applications (BREESE et deKERMADEC 2004b). Les brevets d'invention constituent la mémoire de l'innovation depuis deux siècles (BREESE et deKERMADEC 2004b).

Le brevet a également pour contrepartie l'obligation de la diffusion du contenu scientifique et technique de l'invention, permettant à «l'homme du métier» de pouvoir la reproduire. Le fait que le brevet ne protège pas l'idée (ou la connaissance), mais sa matérialisation permet d'assurer la compatibilité entre l'incitation privée à l'invention (en relevant le rendement privé de l'invention) et le progrès des techniques (en diffusant les connaissances).

Un brevet décrit l'art antérieur et explique en quoi la solution proposée diffère de l'existant, permettant ainsi une excellente traçabilité des choix des conceptions techniques (BREESE et deKERMADEC 2004b).

Depuis la révolution industrielle, brevets et normes techniques s'associent pour promouvoir l'incitation à l'invention (LAPERCHE 2001b).

Il est possible de généraliser le propos tenu sur les brevets d'invention aux informations et connaissances techniques. "Les informations, les connaissances et la Propriété Industrielle forment un ensemble » (BREESE et deKERMADEC 2004b). L'éclairage économique apporte un intéressant point de vue distinctif entre la connaissance et l'information : la connaissance possède quelque chose de plus que l'information, car elle « donne à celui qui la détient une capacité d'action intellectuelle ou physique » (FORAY 2000). «La connaissance est ce qui donne à l'homme des capacités cognitives. L'information, au contraire, reste un ensemble de données, certes formatées et structurées, mais inertes et inactives en ne pouvant pas conférer à celui qui la détient une capacité d'action» (FORAY 2000). 
Formaliser une innovation "à la manière d'un brevet » (deKERMADEC 2001), c'est-à-dire en en décrivant certains aspects avec des documents qui suivent les règles de rédaction des brevets, peut contribuer à renforcer cette vision commune de l'œuvre en train de naître (Dans quel domaine se situe l'invention ? Quel problème veut-on résoudre ? Comment ce problème est-il résolu actuellement ? ...) de façon que, en s'appropriant le style spécifique des brevets d'invention, l'équipe du projet innovant :

- Définisse clairement ses objectifs ;

- S'informe sur l'état de la technique ;

- Imagine une grande variété de solutions ;

- Compare de manière synthétique ses solutions à d'autres ;

- Formalise avec rigueur ce qu'elle veut faire ;

- Communique plus efficacement ;

- Soit davantage consciente que c'est le travail d'équipe qui permet de construire « la meilleure solution » (deKERMADEC 2001).

Formaliser et créer sont donc indissociables (deKERMADEC 2001) : la formalisation «type brevet » est particulièrement adaptée à la démarche inventive. Pierre Breesé et Yann de Kermadec confirment que réinventer coûte cher et qu'il convient de garder la trace des créations de la firme. Utiliser une baseinnovation (c'est-à-dire une base de connaissances dédiée aux procédures et aux produits de l'innovation) permet de mémoriser les idées pour les utiliser au bon moment, d'éviter de réinventer, de repérer des problèmes, des solutions et les personnes à contacter. Elle va contenir des fiches d'innovation, des mémos d'innovation, des brevets, des dossiers d'invention non retenus, ... (BREESE et deKERMADEC 2004b).

Ainsi, le lien entre la propriété industrielle et l'innovation est illustré par le fait que les bases de données des brevets d'invention constituent des sources inégalées et souvent exclusives d'informations technologiques et scientifiques, présentées selon un formalisme puissant du point de vue méthodologique.

\subsection{Liens de l'invention avec les droits de propriété industrielle}

De façon intuitive, il est facile de voir que l'activité inventive nourrit les brevets d'invention. Par ailleurs, la valorisation de la recherche, qui fait partie de la mission des chercheurs, se fait par la concession de licences de brevets sur la base de laquelle différents types de partenariats avec des entreprises industrielles peuvent se monter (BREESE et deKERMADEC 2004b).

\subsection{Liens de l'innovation avec les droits de propriété industrielle}

Là également, de façon intuitive, il est facile de voir que l'innovation, en tant que produit ayant à séduire le marché, nourrit les marques mais aussi les dessins et modèles. Les marques permettent de distinguer un produit ou un service des autres produits et services, tandis que les dessins et modèles permettent de protéger des types de créations esthétiques très variés (BREESE et deKERMADEC 2004b).

\subsection{Liens de l'invention avec l'innovation}

Nous avons vu que la génération d'idées, principale composante de l'activité inventive, relève de ce que la partie gauche de la Figure 2 illustre sous l'appellation de « Front End de l'innovation », pour une démarche d'innovation marchande. C'est donc la partie amont de la procédure d'innovation, celle-là venant nourrir celle-ci.

\subsection{Liens de l'innovation avec l'invention}

De notre point de vue, le produit d'innovation ne peut pas nourrir directement l'activité inventive, du fait, précisément, qu'il se situe à l'aval de la procédure d'innovation et qu'à ce titre il est le résultat d'une connaissance nouvelle mise en action (SAULAIS 2013). 


\subsection{Liens réciproques de l'activité inventive avec le patrimoine intellectuel inventif}

Notre méthode ICAROS® utilise l'analyse du patrimoine intellectuel inventif pour stimuler l'activité inventive (SAULAIS et ERMINE 2016a) : le patrimoine intellectuel inventif de l'organisation contient des éléments de base qui permettent de représenter un certain nombre de trajectoires inventives parcourues par les acteurs des connaissances de l'organisation en reliant les traces intellectuelles inventives laissées (brevets, mémoires de thèse, rapports d'études avancées). Une analyse raisonnée des traces restitue alors le cheminement intellectuel dessiné par le parcours de ces traces (acquisition d'une méthode, approfondissement, interrogation vers une variante, bifurcation, abandon, ...) : on obtient ainsi l'évolution stratégique, au sens de l'entreprise, du patrimoine intellectuel inventif inventorié sur plusieurs années. Le bilan ainsi obtenu par ce procédé, de recueil et d'explicitation des connaissances inventives ayant abouti à une conception nouvelle, est ensuite mis en perspective des connaissances potentielles de chaque domaine (représentant une référence absolue) pour constituer un stimulus cognitif appliqué aux experts représentant chacun leur domaine de connaissances, qui sont individuellement porteurs des éléments prospectifs qu'il faut leur permettre d'identifier, de clarifier pour les aider à élaborer une vision prospective de leur domaine (SAULAIS et ERMINE 2016a).

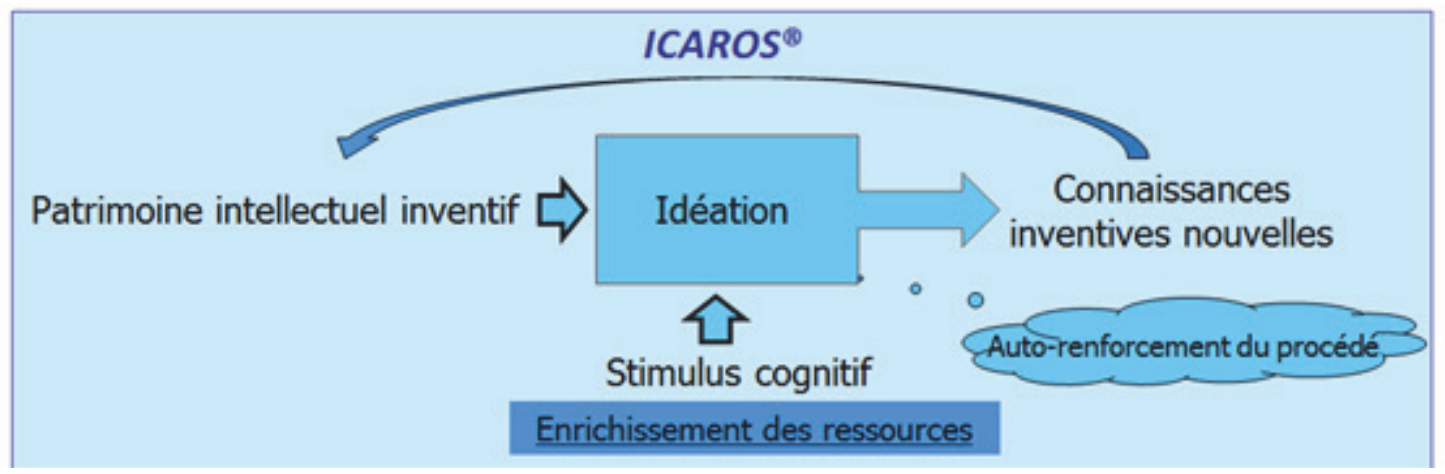

Figure 6. Schéma de production de connaissances inventives par idéation - Source : auteur

La réciproque s'exprime par l'analyse, l'extraction et la mise en action de la connaissance inventive, cette connaissance inventive nouvelle venant enrichir le patrimoine intellectuel inventif (SAULAIS 2016b), (SAULAIS et LECANTE 2016c), comme illustré par la Figure 7, focalisée sur le dossier d'activité inventive dénommé Dossier Invention \& Innovation .

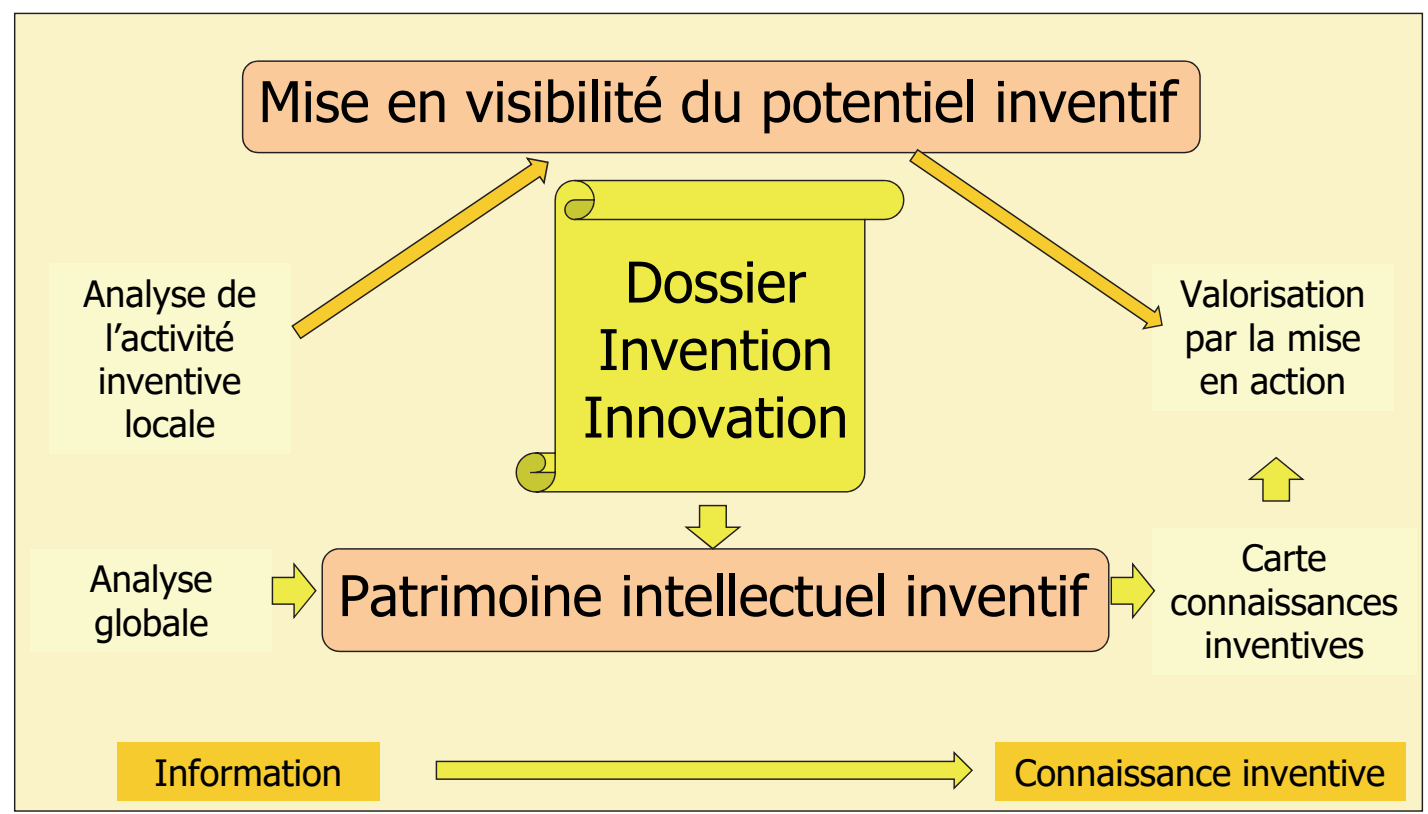

Figure 7 : Analyse de l'activité inventive - Source : auteur 
La Figure 8 illustre les applications de l'analyse de l'activité inventive globale (contenue dans le patrimoine intellectuel inventif) en aide à la décision dans différents domaines.

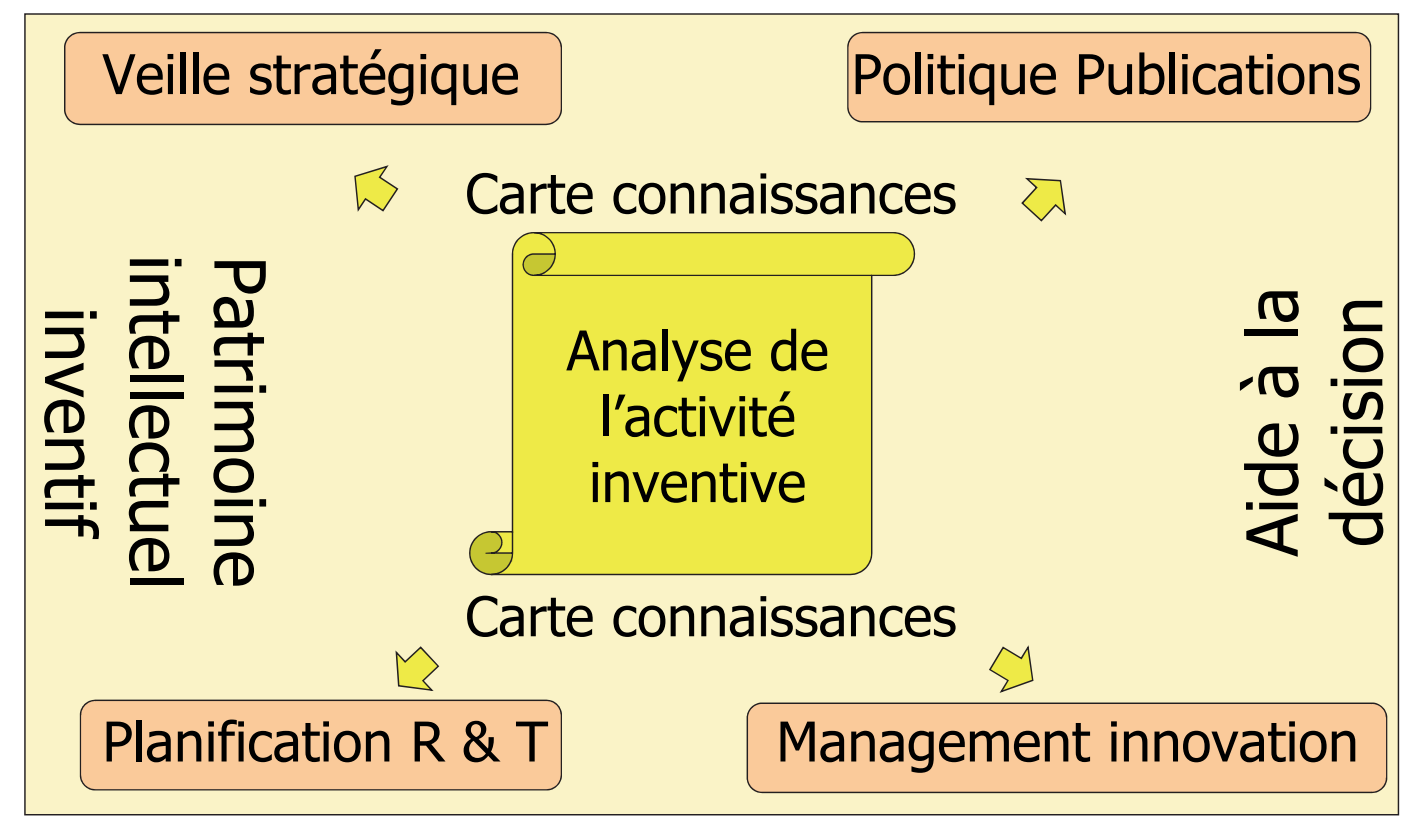

Figure 8 : Applications de la projection de l'analyse de l'activité inventive dans les cartes de connaissances - Source : auteur

\section{La nature des ponts entre les domaines de connaissance}

Notre propos consiste ici à montrer que les liens évoqués précédemment entre deux composants dont l'un nourrit l'autre possèdent une nature beaucoup plus profonde, qui est celle de ponts entre plusieurs domaines de connaissance. Les objets de connaissance que nous avons explorés sont maintenant considérés en tant que représentants d'un domaine de connaissance. Alors, nous pouvons nous interroger sur la nature de ces ponts.

\subsection{Le point de vue des économistes}

Le point de vue des économistes centre son référentiel sur l'organisation : « Comprendre la concurrence, analyser le risque et les stratégies d'appropriation et de commerce, c'est comprendre pourquoi l'innovation et le changement technique résultent de l'apprentissage et de l'accumulation en longues périodes des informations et connaissances scientifiques, techniques et productives. Ainsi posé le problème, les ensembles informationnels de production font entièrement partie du patrimoine industriel et du capital productif de la firme qui les finance et/ou qui se les approprie» (UZUNIDIS 1998). "L'information, surtout celle issue des travaux scientifiques et techniques, est une marchandise [...] c'est un bien-capital qui s'accumule, pour être valorisé, au fil de l'évolution et de l'expansion des rapports marchands [...] La concurrence qui s'aggrave dans le capitalisme industriel a pour conséquence l'appropriation du savoirfaire par l'entreprise et la combinaison d'informations scientifiques et techniques d'origine disciplinaires différente, en ensembles informationnels scientifiques et techniques, détenus par les propriétaires de capitaux » (LAPERCHE 1998).

\subsection{Le point de vue de la gestion des connaissances sur l'innovation}

«L'innovation est l'art de transformer des connaissances en richesses » (BONNAURE 1997) : cette formule de P. Bonnaure fait la synthèse du rôle de la gestion des connaissances dans le « management de l'innovation », puisqu'elle permet d'y voir plus clair (informations à jour, repères communs, traçabilité), de donner du sens à l'action et de nourrir l'esprit d'équipe. 
Soparnot et Stevens précisent que, si l'innovation contribue à régénérer l'organisation en stimulant la création de connaissances, elle en dépend également, puisqu'elle se développe grâce aux connaissances accumulées dans l'organisation : la gestion de l'innovation appelle donc une gestion des connaissances, puisque, pour partie, les connaissances déterminent l'innovation tout comme elles en proviennent (SOPARNOT et STEVENS 2007).

\subsection{Le point de vue de la KBI (innovation fondée sur les connaissances)}

C'est le premier niveau d'application de notre méthode ICAROS® (SAULAIS et ERMINE 2016a). Ce niveau est défini par le fait que la nature des ponts entre les trois domaines de connaissance est caractéristique d'un flux de connaissances, qui va permettre à chaque domaine d'en nourrir un autre (Cf. § 4.7). L'analyse, la cartographie et la mise en action de la connaissance inventive s'inscrivent pleinement dans la montée de la chaîne de valeur de la connaissance, vers la métacognition et la maîtrise décisionnelle dans un monde de plus en plus complexe (SAULAIS et LECANTE 2016c). Une telle démarche, qui reste à instrumenter, permettrait à la firme, vue comme un processeur d'informations, de rendre cohérentes toutes les facettes de son activité stratégique (cf. Figure 8). Cette démarche, développée dans le cadre de la $K B I$, ouvre la voie au domaine de l'IKM (gestion des connaissances inventives ou Inventive Knowledge Management)

\subsection{Le point de vue de l'idéation fondée sur la connaissance}

C'est le deuxième niveau d'application de notre méthode ICAROS® (SAULAIS 2016b), (SAULAIS et LECANTE 2016c), caractérisé par une abstractivité plus grande (donc plus spécifiquement applicable aux deux domaines de connaissance de l'activité inventive et du patrimoine intellectuel inventif). Cette abstractivité nous permet de considérer que l'idéation (au sens de la génération d'idée inventive) au cœur de l'activité inventive est de même nature que son résultat (l'idée inventive, considérée comme une connaissance inventive nouvelle) qui vient lui-même enrichir le patrimoine intellectuel inventif, dont l'analyse et l'extraction de l'activité inventive (de même nature que le résultat qu'elles vont produire) viendront elles-mêmes par leur résultat stimuler l'idéation (Cf. Figure 6) : il s'agit donc d'un phénomène de survenance. De plus, quand l'idée inventive est mise en action dans une œuvre de l'esprit reconnue comme telle par l'octroi de droits de propriété intellectuelle, alors l'inventeur est reconnu comme un auteur (au sens du droit de l'auteur), qui ne fait plus qu'un avec son œuvre puisque l'auteur fait autant l'œuvre que l'œuvre fait l'auteur. Ainsi, la nature du pont entre activité inventive et patrimoine intellectuel inventif (ayant donné lieu à octroi de droits de propriété intellectuelle) est donc la connaissance de la connaissance (ou méta-cognition) :

«C'est la faculté de connaître qui organise la connaissance et non pas les objets qui la déterminent. Le centre de la connaissance est le sujet connaissant : comment notre connaissance reflète-t-elle notre cognition? » (Kant, Emmanuel (1781) La critique de la raison pure)

\section{Conclusion}

Cette étude exploratoire nous a permis de déterminer invention, innovation et droits de propriété intellectuelle comme objets de connaissance, dont notre propos était d'explorer la nature puis les interactions génétiques.

Nous avons ainsi présenté chacun des trois objets de connaissance, tout d'abord l'acte d'innover et son résultat, puis la capacité à inventer, enfin le patrimoine intellectuel inventif et le capital incorporel. En considérant chaque objet de connaissance comme représentant son propre domaine de connaissances, nous avons examiné les liens entre les trois domaines au sens statique et au sens dynamique de l'innutrition de l'un par l'autre. A notre interrogation sur la nature de ces liens, nous avons répondu par la caractérisation de notre méthode $I C A R O S^{\circledR}$ sur deux niveaux d'abstraction: selon le premier niveau, les ponts entre les domaines ont la nature de flux de connaissances inventives transitant d'un domaine à l'autre. Selon le 
second niveau, il s'agit d'un flux engendré par la connaissance de ces connaissances : ce qui nourrit les connaissances inventives d'un domaine (et y permet la création de nouvelles connaissances inventives) est donc l'analyse de la structure de la connaissance déposée dans les connaissances inventives d'un autre domaine.

\section{La bibliographie}

Alland D., Rials, S., Dictionnaire de la culture juridique. Paris: Presses Universitaires de France, 2003.

ALTER N., L'innovation ordinaire. Paris : Eyrolles, 2000.

Altshuller G., Creativity as an Exact science: The Theory of the Solution of Inventive Problem. New York: Gordon and Breach Science Publishing, 1984.

AltShUller G., The Innovation Algorithm: TRIZ, Systematic Innovation and Technocal Creativity. 1998.

AMIDON D., Innovation et management des connaissances. Paris : Editions d'Organisation, 2001.

ARGYRIS C., Savoir pour agir. San Francisco : Jossey-Bass, 1993.

BATESON G., Steps to an Ecology of Mind: Collected essays in Anthropology, Psychiatry, Evolution and Epistemology. Chicago : University of Chicago Press, 1972.

BAUMARD P., Organisations déconcertées. Paris : Masson, 1996.

Benoit-Cervantes G., La Boîte à outils de l'Innovation. Paris : Dunod, 2008.

BIANCHI, L'innovation et ses contraintes : études de cas. Paris : Eyrolles, 1974.

BINCTIN N., Le Capital Intellectuel. Paris: Litec, 2007.

Bloch O., Wartburg W., Dictionnaire étymologique de la langue française. Paris : Presses Universitaires de France, 1932.

BONNAURE P., Futuribles, nº 225 (1997).

BonTIS N., «Intellectual Capital: An exploratory Study that develops Measures and Models » Management Decision 36, $\mathrm{n}^{\circ} 2$ (1998).

BONTIS N., « Managing Organizational Knowledge by Diagnosing Intellectual Capital: Framing and Advancing the State of the Field » International Journal of Technology Management, 1999.

BOUNFOUR A., Capital immatériel, connaissance et performance. Paris, 2006.

Bounfour A., EPINETTE G., Valeur et performance des Systèmes d'Information. Paris : Dunod, 2006.

BOUTILLIER S., «L'entrepreneur face à la propriété industrielle : histoire et actualité » Dans Propriété industrielle et innovation, de Blandine LAPERCHE, 25-48. Paris : L'Harmattan, 2001.

BREESE P., KAISER A., L'évaluation des droits de propriété industrielle. Paris : Gualino, 2004a.

BREESE P., YANN KeRMADEC., La Propriété intellectuelle au service de l'innovation. Paris : Nathan, 2004b.

CALlON M., LATOUR B., «Comment suivre les innovations, clefs pour l'analyse économique » Prospective et santé publique, $\mathrm{n}^{\circ}$ spécial sur l'innovation (10 1985).

Chanal V., "Management de l'innovation de produit industriel : mise en oeuvre d'unedémarche de diagnostic pour améliorer notre compréhension du processus » 7ème conférence de management stratégique. Ecole Centrale de Châtenay, 1999.

CORBEL P., Management stratégique des droits de la propriété intellectuelle. Paris : Gualino, 2007.

CORNU G., Vocabulaire juridique. Paris : Presses Universitaires de France, 1987.

DauZat A., Nouveau dictionaire étymologique et historique. Paris : Larousse, 1971.

DECLERCK R.-P., EYMERY P., CRENER M.-A., Le management stratégique des projets. Paris : Editions Hommes et Techniques, 1980.

KERMADEC Y., Innover grâce au brevet. Paris : Dunod, 2001.

Edvisson L., MALONE M., Intellectual Capital: Realizing Your Company's True Value by Finding its hidden Brainpower. New York : Harper Business, 1997. 
Eltzer T., CAvalluci D., « Parameter Network as a Mean for Driving Problem Solving Process » International Journal of Logistics Management 1, $\mathrm{n}^{\circ} 2$ (2006): 1-10.

FERnEZ-WALCh S., ROMON F., Management de l'innovation. Paris : Vuibert, 2006.

FORAY., D., L'économie de la connaissance. Paris : La Découverte, 2000.

GAFFIOT F., Dictionnaire Latin-Français. Paris : Hachette, 1934.

GARCiA R., CALANTONE R., «A critical look at technological innovation topology and innovativeness terminology: a literature review » The Journal of Product Innovation Management, ${ }^{\circ} 19$ (2001) : 110-132.

GAREL G., MidLER C., « Concourance, processus cognitifs et régulation économique » Revue française de gestion, $\mathrm{n}^{\circ} 104$ (1995) : 86-101.

GiARD V., MiDler C., Pilotage de projets et entreprises. Diversités et convergences. Paris : Economica, 1993.

GuELLEC D., Economie de l'Innovation. Paris : La Découverte, 2009.

HAtchuel A., «Apprentissages collectifs et activités de conception» Revue française de gestion, $\mathrm{n}^{\circ} 99$ (1994) : 109-120.

HATChuel A., WeIL B., « C-K Theory: Notions and Applications of a Unified Design Theory » Herbert Simon International Conference on Design SCiences. Lyon, 2002.

HENDERSON R., CLARK K., "Architectural innovation: the reconfiguration of existing product technologies and the failure of established firms » Administrative Science Quaterly, n 35 (1990) : 9-30.

LAMBERTON D., «Pour une taxinomie de l'information» Dans La Société informationnelle, de Anne MAYERE, 75-84. Paris : L'Harmattan, 1997.

LAMBIN J.-J., Marketing stratégique. New York : MacGraw Hill, 1986.

LAPERCHE B., «Brevets et normes techniques : de l'incitation à l'invention au contrôle de l'innovation » Dans Propriété industrielle et Innovation, de Blandine LAPERCHE, 81-98. Paris : L'Harmattan, 2001b.

LAPERCHE B., La firme et l'information : innover pour conquérir. Paris : L'Harmattan, 1998.

LAPERCHE B., Propriété intellectuelle et Innovation. Paris : L'Harmattan, 2001a.

LE LOARNe S., BIANCO S., Le management de l'innovation. Pearson Education, 2009.

LiTTRE E., Dictionnaire de la Langue Française. Paris: Hachette, 1886.

Louafa T., Perret F.-L., Créativité et innovation. Lausanne : Presses Polytechniques, 2008.

MAFFIN D., «Engineering design models: context, theory and practice » Journal of Engineering Design 9, $\mathrm{n}^{\circ} 4$ (1998) : 315-327.

MARCH J., « Exploration and exploitation in Organizational Learning » Organization Science 1, n 2 (1991).

NAVARRE C., SchaAn J-L., «Où en est la gestion de projet aujourd'hui ? Essai de modélisation » Gestion 2000, n 1 (1989) : 31-57.

NonaKa I., HirotaKa T., La Connaissance créatrice. Bruxelles : De Boeck, 1997.

OCHS, P., « Investisement immatériel et indicateurs de pilotage » Supplément à la revue Echange, n 119 (1996): 60-66.

PRAX J.-Y., BUISSON B., SILBERZAHN P., Objectif Innovation : Stratégies pour co,struire l'entreprise innovante. Paris : Dunod, 2005.

REILLY R., SCHWEIHS R., Valuing Intangible Assets. Irwin Library - McGraw Hil, 2001.

Roos J., Ros G., EdviSSON L., Dragoneti N., Intellectual Capital: Navigating in the New Business Landscape. New York : New York University Press, 1998.

RouQuetTe M.-L., La Créativité. Paris : Presses Universitaires de France, 1973.

SAULAIS P., «Application de la gestion des connaissances à la créativité des experts et à la planification de la R\&T en milieu industriel de haute technologie » thèse de doctorat, Télécom Ecole de Management, 2013.

SAUlais P., « Mise en visibilité du potentiel inventif et représentation du patrimoine intellectuel inventif » Veille Mag, $\mathrm{n}^{\circ} 155$ Spécial Gestion des Connaissances (2016b).

SAULAIS P., LECANTE C., « Analyse, cartographie et mise en action de la connaissance inventive » GeCSO. Paris, $2016 \mathrm{c}$. 
SAULAIS., P., ERMINE J.-L., « Emergence d'innovation technologique incrémentale par aplication, à des experts, de l'analyse du patrimoine intelectuel inventif » 2dité par De Boeck. Innovations 49, nº 1 (2016a) : 103-140.

SCHUMPETER J., Capitalisme, Socialisme et Démocratie. Paris : Payot, 1947.

SENGE P., La cinquième discipline. Paris : General First, 1991.

SIMON H., The Science of the Artificial. Cambridge (MA) : MIT Press, 1969.

SOPARnOt R., STEVEnS E., Management de l'innovation. Paris : Dunod, 2007.

STEWART T., « Brainpower: How Intellectual Capital is Becoming America's Most Valuable Asset» Fortune, 061991.

STEWART T., Intellectual Capital: The new Wealth of Organizations. New York : Doubleday, 1997.

TIDD J., Bessant J., PAVITT K., Management de l'innovation ; intégration du changement technologique, commercial et organisationnel. Bruxelles : De Boeck, 2006.

UZUNIDIS D., «La marchéisation de la recherche publique en France et la question de la propriété industrielle » dans Propriété industrielle et Innovation, de Blandine LAPERCHE, 159-174. Paris : L'Harmattan, 2001.

UZUNIDIS D., «Préface » dans La Firme et l'Information : Innover pour conquérir, de Blandine LAPERCHE, 7-12. Paris : L'Harmattan, 1998.

Vivant M., Bruguiere J.-M., Droit d'auteur. Paris : Dalloz, 2009.

WatZlaWick P., FISCH J., WeaKland R., Changements : Paradoxes et psychothérapies. Paris : Seuil, 1975. 